\title{
Principles and Applications of Magnetic Sensors Based on Magnetic-Sensitive Materials
}

\author{
Kuo Men, Jing Zhu, Ziwei Lian* \\ State Key Laboratory of Advanced Materials for Smart Sensing, GRINM Group Co., Ltd, Beijing, China \\ Email address: \\ menkuo@grinm.com (Kuo Men), zhujing4677@126.com (Jing Zhu), lianziwei@grinm.com (Ziwei Lian) \\ *Corresponding author
}

\section{To cite this article:}

Kuo Men, Jing Zhu, Ziwei Lian. Principles and Applications of Magnetic Sensors Based on Magnetic-Sensitive Materials. Science Discovery. Vol. 6, No. 4, 2018, pp. 283-289. doi: 10.11648/j.sd.20180604.19

Received: June 22, 2018; Accepted: July 19, 2018; Published: August 10, 2018

\begin{abstract}
The core of most magnetic sensors are magnetic-sensitive materials. Magnetic-sensitive materials are sensitive to magnetic field, which could convert magnetic energy into other forms of energy. Magnetic-sensitive materials are including magnetoresistance materials, multiferroic materials, soft magnetic materials and so on. Different magnetic-sensitive materials are applied to different kinds of magnetic sensors, which are used to measure micro-magnetic fields under different application environments and precision requirements. The performance of magnetic-sensitive materials has become the limiting factor for the development of such magnetic sensors. In this paper, the principle and application of magnetic sensors based on magnetic sensitive materials are described.
\end{abstract}

Keywords: Magneto-Sensitive Materials, Magnetic Sensor, Energy Transformation

\section{基于磁敏材料的磁场传感器的原理及应用}

门阔, 朱婧, 连紫薇"

北京有色金属研究总院, 智能传感功能材料国家重点实验室, 北京, 中国

邮箱

menkuo@grinm.com（门阔）, zhujing4677@126.com(朱婧), lianziwei@grinm.com(连紫薇)

摘要: 磁场传感器种类繁多, 其中大部分磁场传感器核心材料都是磁敏材料, 而磁敏材料是指对磁场敏感的一类磁性 材料, 其作用在于将磁能量转换成其他形式能量, 磁敏材料包括磁电阻材料、多铁性材料、软磁材料等多种磁性材料。 不同磁敏材料应用于不同种类磁场传感器, 这类传感器用以对不同应用环境、不同精度要求条件下的微磁场测量。磁 敏材料的性能成为了这类磁场传感器未来发展的制约因素。本文阐述了各类基于磁敏材料的磁场传感器的原理及应用, 并对未来发展进行了讨论和展望。

关键词: 磁敏材料, 磁场传感器, 能量转换

\section{1. 引言}

随着现代科技的进步，微磁场测量技术的应用越来越 广泛, 尤其在空间定位、医疗监测、矿物勘探、电力检测
等领域增长迅速, 从最古老的霍尔传感器到迄今为止最精 确的的超导量子干涉仪, 磁场测量精度已经提升了十几个 数量级, 应用范围大大增加, 从原理上讲基本可以达到地 磁场微扰测量和生物体弱磁信号等微磁场测量的应用条 件, 但一些复杂条件下的实际应用仍有待进一步提高。因 
此迫使磁场传感器不断向着高灵敏度、高分辨率、小型化 以及和电子设备兼容的方向发展。

各类磁场传感器工作原理不尽相同, 但其实质都是将 磁信号转换为更方便测量的信号, 完成这一能量转换过程。 常见的磁场传感器及检测精度如图所示。
其中诸如超导量子干涉仪、光泵浦等磁场传感器工作 过程不依赖于磁敏材料。

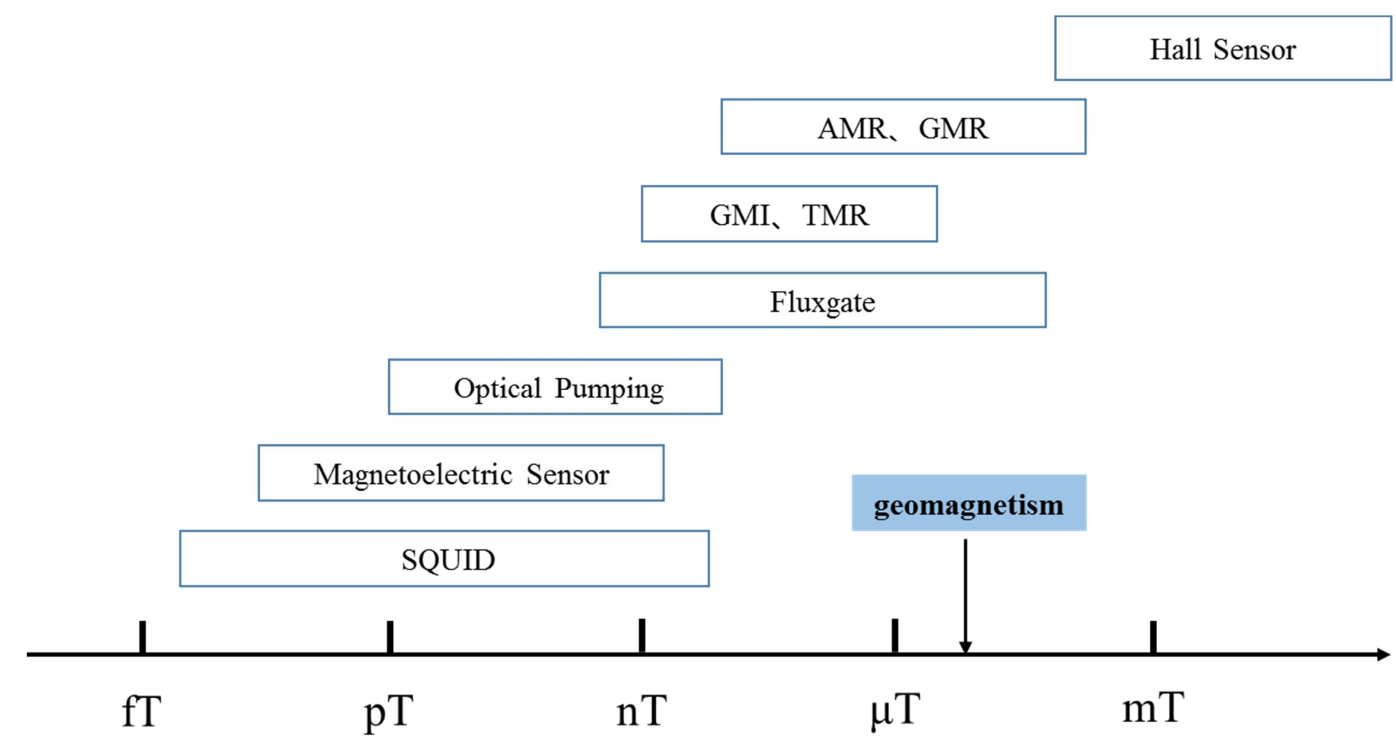

图1 各类磁场传感器测量范围。

超导量子干涉仪实质是一种将磁通转化为电压的磁 通传感器, 其基本原理是基于超导约瑟夫森效应和磁通量 子化现象。超导量子干涉中两块超导体被一薄势垒层分开, 构成一个约瑟夫森隧道结。当含有约瑟夫森隧道结的超导 体闭合环路被适当大小的电流偏置后, 会呈现一种宏观量 子干涉现象, 即隧道结两端的电压是该闭合环路环孔中的 外磁通量变化的周期性函数, 其周期为单个磁通量子 $\Phi 0=2.07 \times 10-15 \mathrm{~Wb}$, 这样的环路就叫做超导量子干涉仪 $[1]$ 。

光不浦高灵敏度磁场测量技术已经有超过半个世纪 的历史, 该技术由H.G.Dehmelt[2]和 W.E.Bell等人[3]开创, 后经 C.Cohen-Tannoudji等人[4]进一步发展。该方法的基 本原理是使用与光学共振跃迁接近的激光来光泵浦基态
原子，使其产生长寿命取向和/或更高阶矩，激发态原子 随后在待测磁场中进行自旋进动。该进动改变了原子的光 吸收和色散特性, 在外磁场作用下原子自旋进动频率产生 线性变化, 通过光探测器检测光谱频移来获得被测磁场 [5]。

相比上述两类传感器, 霍尔磁场传感器、磁电阻传感 器、多铁性薄膜磁场传感器以及磁通门传感器等类型磁场 传感器则依赖于各类磁敏材料。

磁敏材料是一类磁性材料的统称, 顾名思义是指对磁 场敏感的材料, 其作用在于将磁信号转换成其他形式能量 的材料, 包括磁电阻材料、多铁性材料、软磁材料等。本 文将对各类基于磁敏材料的磁场传感器原理及应用进行 阐述。

\section{2. 各类基于磁敏材料的磁场传感器原理及应用}

\section{1. 霍尔传感器}

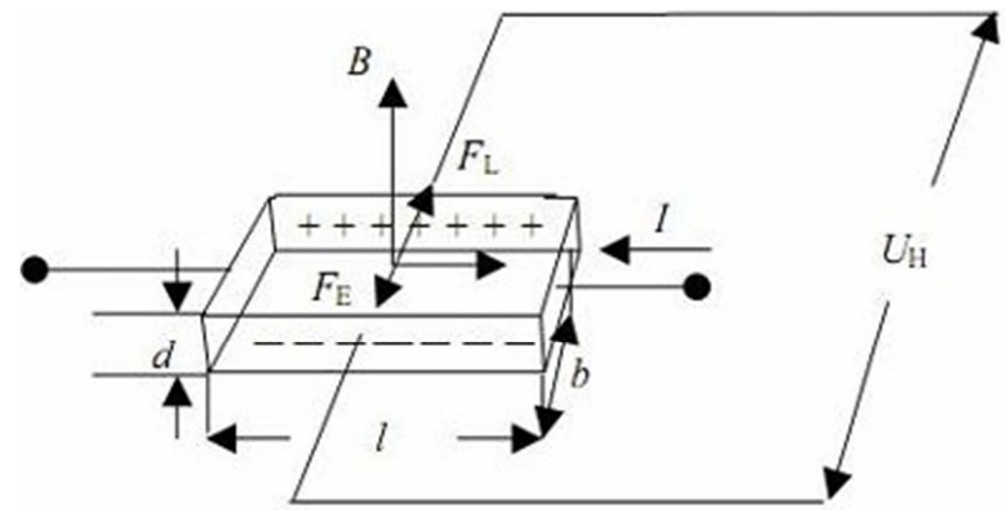

图2 霍尔传感器原理图。 
霍尔传感器是根据霍尔效应制作的一种磁场传感器 [6]。霍尔效应是磁电效应的一种, 是霍尔于 1879 年在研究 金属导电机理时发现的。霍尔效应描述了运动的带电粒子 在磁场中受洛仑兹力作用而引起偏转, 当带电粒子被约束 在固体材料中, 这种偏转就导致在垂直电流和磁场的方向 上产生正负电荷的聚积, 从而形成附加的横向电场。电荷 在横向受力为零时不再发生横向偏转, 结果电流在磁场作 用下在器件的两个侧面出现了稳定的异号电荷堆积从而 形成横向霍尔电场:

$$
\mathrm{E}=-\frac{v B}{c}
$$

制造霍尔器件的半导体材料主要是Ge、Si、GaAs、InAs、 $\mathrm{InSb}$ 等。这类半导体材料虽然不属于磁性材料, 但相比金 属而言, 在磁场中可以观测到很强的霍尔效应, 因此将其 归类为“磁敏材料”。霍尔器件一般用 $\mathrm{N}$ 型材料, 因为电子 迁移率比空穴的大得多, 器件可以有较高的灵敏度。除了 用整块半导体材料做霍尔器件外, 还可以用薄膜制作霍尔 器件。在绝缘祄底上淀积薄膜或用外延或离子注入等方法 在高电阻率的半导体祄底上制造一层厚度为微米量级的 薄膜。用离子注入或处延法制造的砷化镓霍尔器件在很宽 的磁场强度范围内有很好的线性关系, 并且能在很宽的温 度范围内稳定地工作。用硅外延或离子注入方法制作的薄 膜霍尔器件可以和集成电路工艺兼容, 广泛应用于智能手 机、平板电脑和导航设备等移动终端, 拥有巨大的市场前 景工艺。该类器件相对一般IC更为简单, 通过金属电极将 传感器与其他电路 (如放大器、调节处理器等) 相连。增 加了磁通集中器 (工艺上来讲就是做原来的管芯上增加一 层坡莫合金) 的霍尔磁力计实现了从单轴到三轴磁力计的 跨越式发展。同时, 霍尔磁力计可以与加速度计组成六轴 电子罗盘, 三种惯性传感器 (加上陀螺仪) 组合在一起还 能实现九轴组合传感器, 构成更强大的惯性导航产品。

霍尔传感器具有以下优点:

1. 霍尔传感器可以测量任意波形的交变磁场, 甚至对 瞬态峰值的测量。副边电流忠实地反应原边电流的 波形。而普通互感器则是无法与其比拟的;

2. 原边电路与副边电路之间有良好的电气隔离;

3. 精度高: 在工作温度区内精度优于 $1 \%$, 该精度适 合于任何波形的测量;

4. 线性度好：优于 $0.1 \%$;

\section{2. 磁电阻传感器}

磁电阻, 即磁致电阻, 是指电阻率 $\rho$ 在外加磁场 $\mathrm{H}$ 的作 用下所产生的变化。目前已成功商业化应用的磁电阻传感 器一般分为三类: 各向异性磁电阻 (AMR) 传感器、巨 磁电阻 (GMR) 传感器、隧道磁电阻 (TMR) 传感器[7]。

\subsection{1. 各向异性磁阻（AMR）传感器}

某些材料的电阻值会随着外加磁场的大小发生变化, 这种现象叫做磁阻效应, 磁阻传感器利用磁阻效应制成。

1857年, Thomson发现坡莫合金的各向异性磁阻效应。 即磁阻的变化与磁场和电流间夹角有关 $[8]$ 。
当外部磁场与磁体的内建磁场有一定角度的时候, 磁 体内部磁化矢量会偏移, 薄膜电阻降低, 我们对这种特性 称为各向异性磁电阻效应 (Anisotropic Magnetoresistive Sensor，简称AMR）。磁场作用效果如下图。

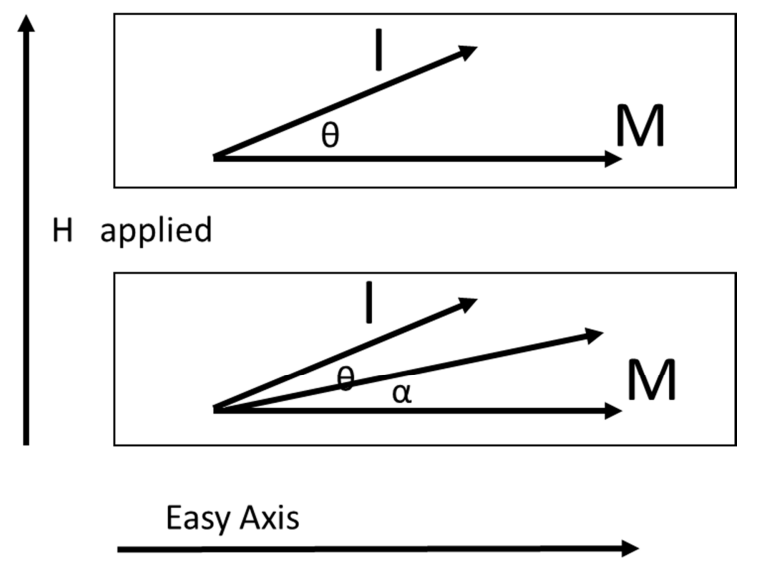

图3 AMR磁场效果图。

AMR磁传感器的基本结构由四个磁阻组成了惠斯通 电桥。当在该电路上施加一个偏置磁场时, 两个相对放置 的电阻的磁化方向就会朝着电流方向转动, 这两个电阻的 阻值会增加; 而另外两个相对放置的电阻的磁化方向会朝 与电流相反的方向转动, 该两个电阻的阻值则减少。通过 测试电桥的两输出端输出差电压信号, 可以得到外界磁场 值。

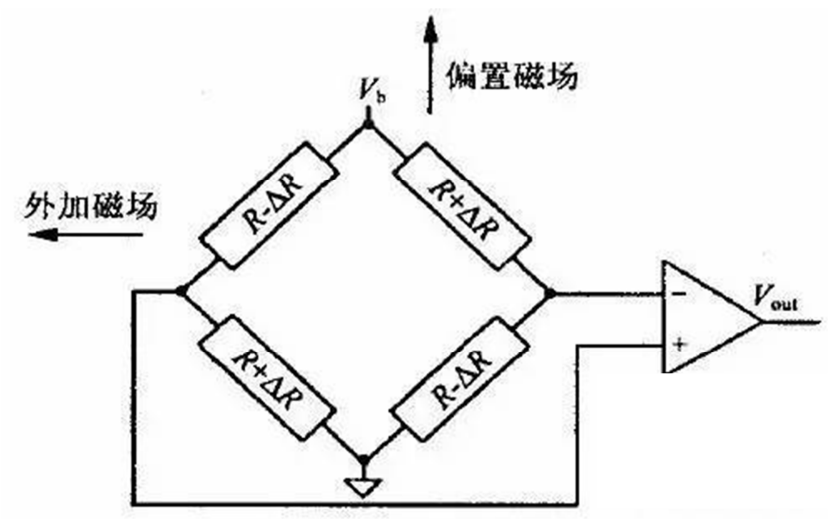

图4 AMR传感器原理图。

各向异性磁阻（AMR）技术的优势有以下几点:

1. 各向异性磁阻（AMR）传感器具有广大的运作空 间, 无需像霍耳元件那样增加聚磁等辅助手段。

2. AMR技术核心是一层磁性薄膜, 工艺相对较为简 单, 成本低, 不需要昂贵的制造设备。

3. AMR技术具有高频、低噪和高信噪比特性, 在各 种应用中尚无局限性。

AMR磁阻传感器可以很好地感测地磁场范围内的弱 磁场测量, 广泛应用于导航罗盘、各种磁卡机、旋转位置 传感、电流传感、偏航速率传感器和虚拟实景中的头部轨 迹跟踪器。 
AMR传感器的核心材料是各向异性磁敏材料, 目前 一般采用坡莫合金, 坡莫合金的晶粒尺寸及形貌对传感器 性能起着重要影响。

\subsection{2. 巨磁阻（GMR）传感器}

1988年，法国科学家费尔在 $(\mathrm{Fe} / \mathrm{Cr})$ 相间的多层膜 电阻中发现: 微弱的磁场变化可以导致电阻大小的急剧变 化, 其变化的幅度比通常高十几倍[9]。

一般的磁铁金属, 在加磁场和不加磁场下电阻率的变 化为 $1 \%$ \% $\%$ ，但铁磁金属/非磁性金属/铁磁金属构成的多 层膜, 在室温下可以达到 $25 \%$, 低温下更加明显, 这也是 巨磁阻效应的命名缘由。

常规磁电阻源于磁场对电子运动的直接作用, 呈各向 异性磁阻, 即电阻与磁化强度和电流的相对取向有关。相 反, GMR磁阻呈各向同性, 与磁化强度和电流的相对取 向基本无关。

GMR效应的首次商业化应用是 1997年, 由IBM公司投 放市场的硬盘数据读取探头。到目前为止，巨磁阻技术已 经成为全世界几乎所有电脑、数码相机、MP3播放器的标 准技术。

具有GMR效应的材料主要有多层膜、颗粒膜、纳米 颗粒合金薄膜、磁性隧道结合氧化物、超巨磁电阻薄膜等 五种材料。其中自旋阀型多层膜的结构在当前的GMR磁 阻传感器中应用比较广泛。

自旋阀主要有自由层（磁性材料FM）, 隔离层（非 磁性材料 $\mathrm{NM}$ ), 钉扎层 (磁性材料FM) 和反铁磁层 ( $\mathrm{AF}$ ) 四层结构。

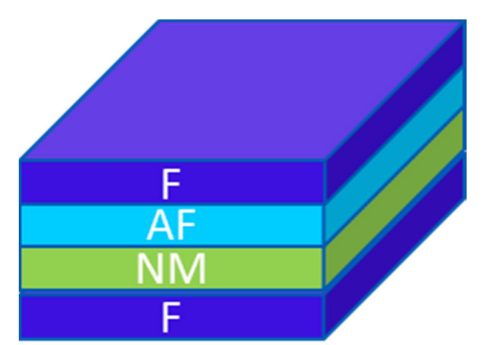

图5 自旋阀GMR磁阻传感器基本结构。

同AMR传感器类似, GMR磁阻传感器也具有惠斯通 电桥结构, 该结构可以减少外界环境对传感器输出稳定性 的影响, 增加传感器灵敏度。当相邻磁性层磁矩平行分布, 两个FM/NM界面呈现不同的阻态, 一个界面为高阻态,

一个界面为低阻态, 自旋的传导电子可以在晶体内自由移 动, 整体上器件呈现低阻态; 而当相邻磁性层磁矩反平行 分布, 两种自旋状态的传导电子都在穿过磁矩取向与其自 旋方向相同的一个磁层后, 遇到另一个磁矩取向与其自旋 方向相反的磁层, 并在那里受到强烈的散射作用, 没有哪 种自旋状态的电子可以穿越FM/NM界面, 器件呈现高阻 态。GMR磁阻传感器商业化时间晚于霍尔传感器和AMR 磁阻传感器, 制造工艺相对复杂, 生产成本也较高。但其 具有灵敏度高、能探测到弱磁场且信号好, 温度对器件性 能影响小等优点, 因此市场占有率呈稳定状态。GMR磁 阻传感器在消费电子、工业、国防军事及医疗生物方面均 有所涉及。

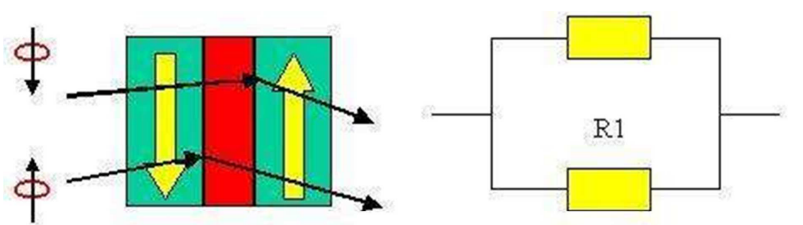

反铁磁耦合时 (外加磁场为0) 处于高阻态的导电输运特性, 电阻: R $1 / 2$
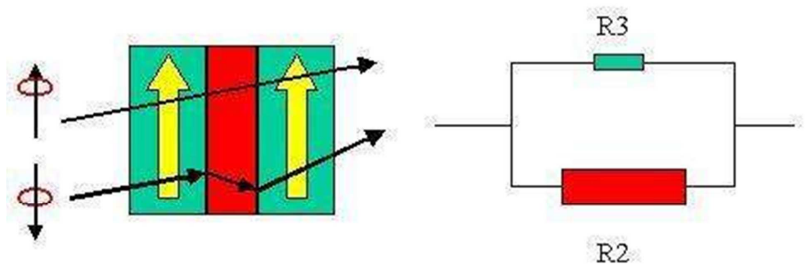

外加磁场使该磁性多层薄膜处于饱和状态时( 相邻磁性层磁矩 平行分布, 而电阳处于低阻态的导电输运特性, 电阻: R $2 *$ R 3 / (R2+R3)，R2>R1>R3

图6 自旋阀GMR磁阻电路图。

\subsection{3. 隧道磁阻（TMR）传感器}

早在 1975 年, Julliere 就在 $\mathrm{Co} / \mathrm{Ge} / \mathrm{Fe}$ 磁性隧道结 (MagneticTunnelJunctions, MTJs) 中观察到了 TMR (Tunnel Magneto-Resistance) 效应[10]。但是, 这一发现 当时并没有引起人们的重视。在此后的十几年里，有关 TMR 效应的研究进展十分缓慢。在GMR效应的深入研究 下, 同为磁电子学的 TMR效应才开始得到重视。2000年, $\mathrm{MgO}$ 作为隧道绝缘层的发现为 TMR磁阻传感器的发展契 机。

2008年，日本东北大学的S. Ikeda，H. Ohno团队[11] 实验发现磁性隧道结 $\mathrm{CoFeB} / \mathrm{MgO} / \mathrm{CoFeB}$ 的电阻率变化在 室温下达到 $604 \%$, 在 $4.2 \mathrm{~K}$ 温度下将超过 $1100 \%$ 。 TMR 效 应具有如此大的电阻率变化, 因此业界越来越重视TMR 效应的研究和商业产品开发。

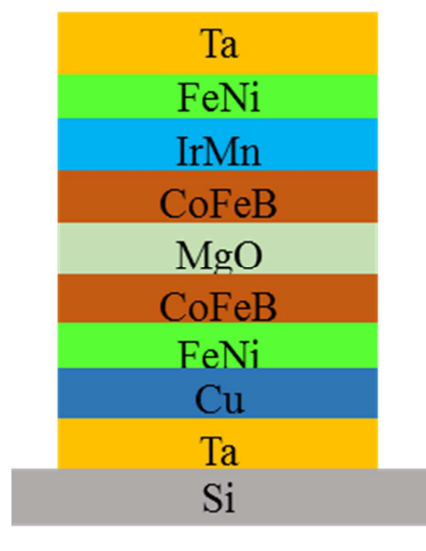

图7 TMR磁阻传感器基本结构。

TMR元件在近年才开始工业应用的新型磁电阻效应 传感器, 其利用磁性多层膜材料的隧道磁电阻效应对磁场 进行感应，比之前所发现并实际应用的AMR元件和GMR 元件具有更大的电阻变化率。 

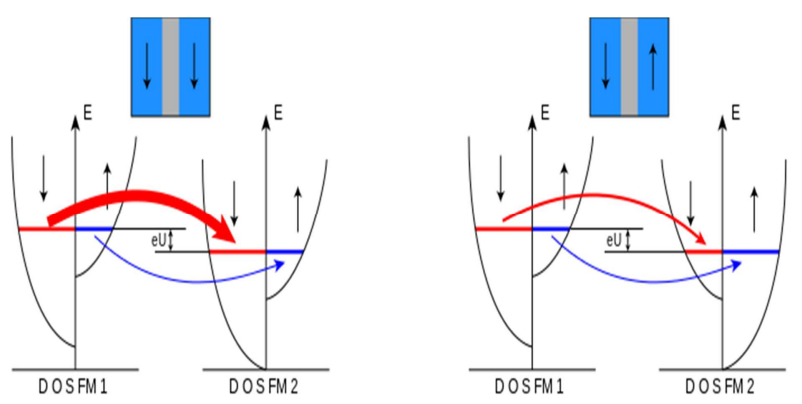

图8 TMR磁阻传感器的材料结构及原理。

当两层铁磁层的磁化方向互相平行, 多数自旋子带的 电子将进入另一磁性层中多数自旋子带的空态, 少数自旋 子带的电子也将进入另一磁性层中少数自旋子带的空态, 总的隧穿电流较大, 此时器件为低阻状态; 当两层的磁铁 层的磁化方向反平行, 情况则刚好相反, 即多数自旋子带 的电子将进入另一磁性层中少数自旋子带的空态, 而少数 自旋子带的电子也进入另一磁性层中多数自旋子带的空 态, 此时隧穿电流较小, 器件为高阻状态。

可以看出, 隧道电流和隧道电阻依赖于两个铁磁层磁 化强度的相对取向, 当磁化方向发生变化时, 隧穿电阻发 生变化, 因此称为隧道磁电阻效应。

TMR传感器具有以下优点:

1. 温度稳定性好;

2. 更高的灵敏度;

3. 低功耗;

4. 不需要额外的聚磁环结构和 set/reset线圈结构。

目前, 商业化 TMR 器件所用材料体系多为 $\mathrm{CoFeB} / \mathrm{MgO} / \mathrm{CoFeB}$, 其制备关键在于超薄 $\mathrm{MgO}$ 势垒层的 控制, 商业化的 TMR 器件一般只用磁控溅射的方法制备多 层膜, 势垒层厚度需精确控制到 $0.1 \mathrm{~nm}$, 这使其制备难度 大大增加, 并对该类此磁控㳚射设备提出了很高的要求。 目前世界上只有希捷等少数公司掌握该技术, 并具备自主 知识产权。另外, 全钲钛矿结构 (如 $\mathrm{LaSrMnO}$ ) 的 TMR 器件是目前研究的热点, 提高其居里温度, 是该类材料 TMR商业化的需要解决的重要问题。

\section{3. 磁通门传感器}

从1931年，第一项磁通门方面的专利被授予 H.P.Thomas[12]开始, 磁通门就在导航、磁测量等领域快 速发展 [13-14]。最常见的是磁通门在导航系统中作为罗盘 的应用。同时, 作为磁传感器, 磁通门在航空航天以及空 间探测领域也得到了广泛应用[15]。

磁通门传感器的核心材料是软磁材料, 利用被测磁场 中高导磁铁芯在交变磁场的饱和激励下, 其磁感应强度与 磁场强度的非线性关系来测量弱磁场的一种传感器。磁通 门传感器的基本原理是基于铁芯材料的非线性磁化特性, 其敏感元件为高磁导率、易饱和材料制成的铁芯, 有两个 绕组围绕该铁芯: 一个是激励线圈, 另一个是信号线圈。 在交变激励信号的磁化作用下, 铁芯的导磁特性发生周期 性饱和与非饱和的变化, 从而使围绕在铁芯上的感应线圈 感应出反应外界磁场的信号, 该信号可以通过特定的检测 电路将其提取出来。

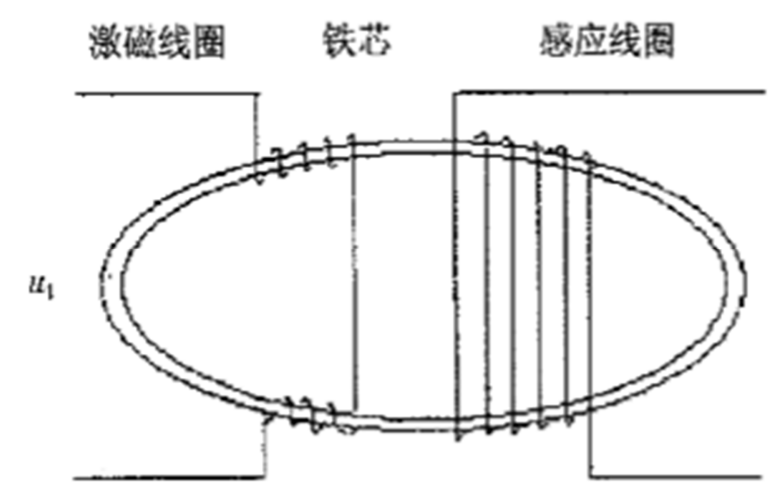

图9 磁通门传感器结构及原理。

磁通门作为一种具有优良综合性能的弱磁场测量器 件, 与霍尔器件、磁阻器件等磁测量器件相比较在灵敏度、 分辨率、温度稳定性、零位漂移等方面具有优势 [16], 然 而其存在的缺点是体积大, 将磁通门的铁芯和线圈通过微 加工工艺制备在硅或其他基底上制成的微型磁通门可以 解决磁通门体积和质量过大的问题。图 10 为最简单的 MEMS磁通门器件。[17]

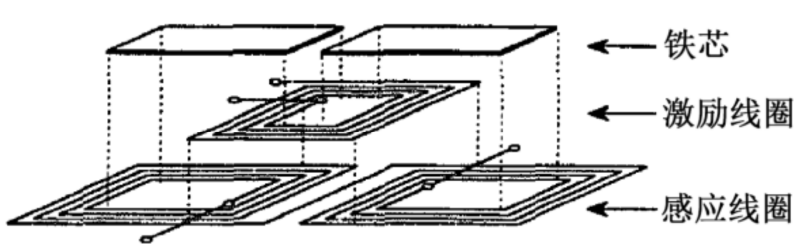

图10 MEMS磁通门传感器结构图。

MEMS磁通门具有很多优点:

1. 基于MEMS工艺的大规模生产能降低单个磁通门 的成本;

2. 微型磁通门的铁芯尺寸小更利于磁通门工作在高 频状态、提高磁通门的频率范围;

3. 批次性误差和装配误差能得到极好的控制, 这使得 微型磁通门在多轴或磁通门阵列中展现出更大的 优势。

当然, MEMS磁通门传感器仍存在很多问题:

1. 功耗问题。磁通门工作所需要的激励电流不会随着 磁通门体积的减小而大幅减小, 也就是说功耗不会 大幅降低。而因功耗释放出的热量集中在很小的区 域将对传感器及由其组成的片上系统的热稳定性 造成威胁, 这会给磁通门的噪声、稳定性以及寿命 等带来不利影响;

2. 微型正交激励磁通门的噪声和灵敏度问题。灵敏度 低是由于铁芯横截面积较小和铁芯磁性能较差导 致的，而灵敏度过低本身就会导致噪声高;

3. 采用微加工工艺所制备铁芯性能较差问题。通过磁 控溅射、电镀等方式制备的铁芯材料磁性能与传统 磁通门所用带材性能相比具有明显差距, 这使得微 型磁通门各项性能均受到影响;

4. 软磁薄膜性能问题。传统的磁通门传感器用的软磁 材料是块体材料, 而MEMS器件采用薄膜材料, 目 前一般采用磁控溅射的方法制备软磁薄膜, 该方法 
制备的薄膜与三维块体材料成分及组织结构上存 在很大差异, 尤其需要增强其非晶形成能力, 以便 得到更好的非晶或纳米晶软磁薄膜。

\section{4. 多铁性材料磁场传感器}

多铁性材料是指材料中包含两种及两种以上铁的基 本性能, 这些铁的基本性能包括铁电性（反铁电性）, 铁 磁性（反铁磁性、亚铁磁性）和铁弹性。是一种集电与磁 性能于一身的多功能材料。

多铁性材料可以分为以下类型[18]:

1. 单相多铁性。研究最多、用途最广的铁电材料是 $\mathrm{ABO}_{3}$ 钙钛矿结构氧化物。在具有这种结构的铁电 材料中, 铁电性多数来源于位于氧八面体中心的 $\mathrm{B}$ 位离子, 在居里温度以下偏离了氧八面体中心, 降 低了晶体结构的对称性, 使正负电荷中心分离形成 电偶极矩。如 $\mathrm{BiFeO}_{3}, \mathrm{BiMnO}_{3}, \mathrm{PbFe}_{1 / 2} \mathrm{Nb}_{1 / 2} \mathrm{O}_{3}$; 稀土锰氧化物, 如六方 $\mathrm{RMnO}_{3}(\mathrm{R}=\mathrm{Sc}, \mathrm{Y}, \mathrm{In}$, $\mathrm{Ho}, \mathrm{Er}, \mathrm{Tm}, \mathrm{Yb}, \mathrm{Lu}$ ），以及正交结构的 $\mathrm{RMn}_{2} \mathrm{O}_{5}$ $(\mathrm{R}=\mathrm{Y}, \mathrm{Tb}, \mathrm{Dy}, \mathrm{Ho})$; 方硼石, 化学式为 $\mathrm{M}_{3} \mathrm{~B}_{7} \mathrm{O}_{13} \mathrm{X}$ $(\mathrm{M}=\mathrm{Cr}, \mathrm{Mn}, \mathrm{Fe}, \mathrm{Co}, \mathrm{Cu}, \mathrm{Ni}, \mathrm{X}=\mathrm{Cl}, \mathrm{Br}$ ) ; $\mathrm{BaMF}_{4}$ 化合物 $(\mathrm{M}=\mathrm{Mg}, \mathrm{Mn}, \mathrm{Fe}, \mathrm{Co}, \mathrm{Ni}, \mathrm{Zr}$ ) 等[19]。

2. 复合陶瓷材料。目前, 通过高温共烧方法可以制备 出多种铁电氧化物与磁性氧化物共存的复相陶瓷 材料, 但仍存在诸多问题, 高温烧结过程中两陶瓷 相之间共烧失配、原子互扩散与反应等, 使得高温 共烧复合陶瓷的磁电系数并不高。而降低陶瓷烧结 温度又会带来烧结不完全、致密度低的缺点, 也会 影响材料的性能。近来, 为了在避免互扩散的同时 提高烧结密度, 新的烧结技术 (如放电等离子烧结 SPS、微波烧结等) 被用来制备复合陶瓷。

3. 铁磁合金基巨磁电复合材料。合金基磁电复合材料 的制备是将合金与铁电材料直接粘接而成, 制备非 常简单。由于Terfenol-D 材料的起始磁导率相对较 低, 而饱和场较高, 使Terfenol-D基磁电复合材料 不适合在低磁场中使用。选用一些软磁合金, 如 $\mathrm{Ni}(\mathrm{Mn}-\mathrm{Ga}), \mathrm{Ni}$, 非晶态合金等[20], 可以显著改 善复合材料在低磁场下的性能 [21]。近来, 为了避 免两相之间有机粘接层对材料性能的影响, 可以将 磁性金属通过磁控溅射或电化学方法直接沉积在 压电材料表面，以消除两相之间的额外的粘接层 [22]。

4. 高分子基复合材料。在金属合金基叠层复合材料中, 导电金属合金在高频下具有较大的涡流损耗。以绝 缘高分子作为基体可降低复合材料的高频浴流损 耗，同时高分子既作为基体又作为黏结剂。尽管高 分子相作为基体会降低压电和/或铁磁相的含量而 影响复合材料磁电性能, 但相比于陶瓷复合材料和 金属合金基复合材料，高分子基磁电复合材料仍 具有独特的优势。采用高分子作为基体固化, 避免 了陶瓷材料的高温烧结, 其低温制备工艺简单, 成
本较低; 同时具有出色的工艺性能, 能够根据需要 方便地加工成各种特定形状。

磁电效应就是多铁性材料中一种由铁电和磁性相互 作用而产生的耦合效应。利用磁电效应制成的传感器可用 于磁场探测, 原理简单而直接[23]。由于在磁电系数测试 中需要施加直流偏置磁场 $\mathrm{H}_{\mathrm{dc}}$ 和交流微扰磁场 $\mathrm{H}_{\mathrm{ac}}$, 这样, 对磁电系数已确定的磁电复合薄膜, 已知 $\mathrm{H}_{\mathrm{dc}}$ 或 $\mathrm{H}_{\mathrm{ac}}$ 时即可 探测出另一个磁场分量。材料的磁电系数越大, 对磁场的 灵敏度越高。测试原理图如图所示。

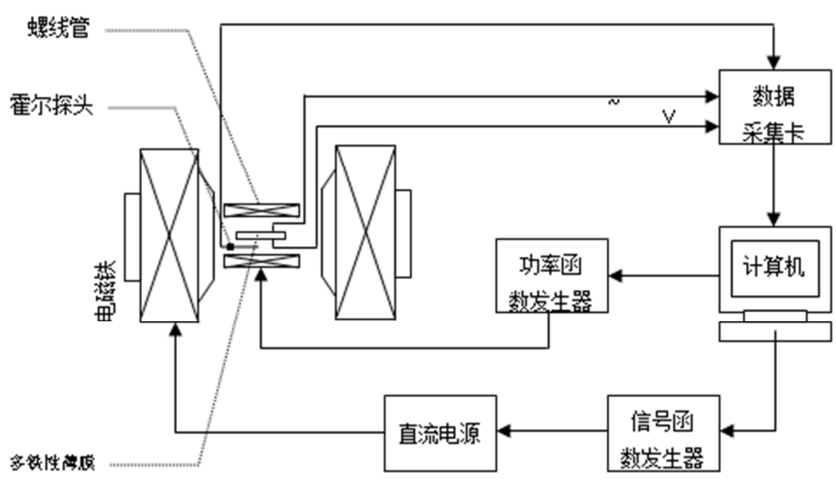

图11 利用多铁性薄膜进行磁场检测原理图。

该类型测试台本质上是为测磁电耦合系数而搭建的 [24], 磁电耦合系数 $\alpha_{\mathrm{E}}=\mathrm{dE} / \mathrm{dH}$, 而在磁场测量过程中, 若 材料 $\alpha_{\mathrm{E}}$ 已知, 即可求出待测磁场 $\mathrm{H}$ 。

在交流磁场探测方面, 弗吉尼亚理工大学研究小组 [25]以高品质因数的PMN-PT单晶与Terfenol-D粘接制得 三层磁电复合材料, 在一定偏置磁场下对样品施加微弱的 交变磁场, 使用锁相放大器测试样品两端产生的磁电电压。 材料磁电电压响应与交流磁场幅值基本成线性关系, 经良 好的屏蔽处理, 材料在谐振频率下可以探测到 $1.2 \times 10^{-10} \mathrm{~T}$ 的交流磁场。在直流磁场探测方面, 由于磁电复合材料的 磁电响应会随着直流磁场的变化而发生改变, 当给材料施 加固定频率和幅值的交流磁场时, 即可探测出直流磁场沿 材料测试方向的大小。若将三个相同的磁电复合材料正交 排列, 还可以标定出空间磁场的方向。以Terfenol-D/PZT 叠层复合材料为例, 通过绕在复合材料外的线圈对复合材 料施加 $0.10 \mathrm{e}$ 的交流磁场, 从而测试了其对直流磁场的响 应[26]

从材料角度讲, 对于基于磁电复合材料的磁传感器, 一般来说, 材料的磁电系数越大, 器件的灵敏度就越高。 但对于整体器件设计而言角, 无处不在的噪音信号会显著 降低器件的灵敏度, 因此除了提高材料磁电系数, 为满足 实际应用还应提高器件的信噪比[27]。

\section{3. 结论}

磁敏材料的发展制约着各类磁场传感器的发展, 因此 掌握核心磁敏材料的制备技术是各类磁场传感器走向商 业化的重要步骤。

用于磁场传感器的磁敏材料多为薄膜材料, 该类磁敏 薄膜制备过程中成分及形貌的控制直接影响材料性能, 从 
而影响磁场传感器灵敏度, 因此可靠地薄膜制备技术是该 类传感器商业化生产的基础。

多铁性磁场传感器所用的多铁性材料、磁通门传感器 所用的软磁材料都是从三维材料演变而来, 但在二维材料 制备问题上与三维材料存在很大差异,一是由于各类元素 功函数不同, 溅射所用靶材成分与薄膜差异较大, 为得到 成分精确的薄膜, 需对靶材成分进行优化。二是制备过程 中二维材料的形貌与祄底结构、制备温度等多因素相关, 其形核长大机理不同于三维材料, 因此在取向生长、晶粒 尺寸控制等问题上需注意。

磁电阻传感器所用多层膜材料制备过程更为苛刻, 由 于磁电阻多层膜间各类磁相互效应对薄膜厚度敏感, 因此 需严格控制薄膜厚度及均匀性, 这也对制备设备提出了较 高的要求。

未来更多新型磁敏材料的诞生以及新的材料制备技 术的出现, 会直接驱动磁场传感器的发展。

\section{致谢}

本研究是在北京有色金属研究总院科技创新基金支 持下完成。在此表示感谢!

\section{参考文献}

[1] 陈林,李敬东,唐跃进,等.超导量子干涉仪发展和应用现状 [J]. 低温物理学报,2005,27(s1):657-661。

[2] Dehmelt H G. Modulation of a Light Beam by Precessing Absorbing Atoms[J]. Physical Review, 1957, 105(6):1924-1925.

[3] Bell W E, Bloom A L. Optically Driven Spin Precession[J]. Physical Review Letters, 1961, 6(6):280-281.

[4] Cohentannoudji C, Dupontroc J, Haroche S, et al. Detection of the Static Magnetic Field Produced by the Oriented Nuclei of Optically Pumped 3He Gas[J]. Physical Review Letters, 1969, 22(15):758-760.

[5] 张鹏,陈洪娟,桂永雷,等.基于MEMS技术的光泵原子磁力仪 发展与应用 $[\mathrm{J}]$.微纳电子技术, 2017,54(7):465-471。

[6]曲宏泽. 霍尔效应磁敏传感器及其应用 [J]. 自动化仪 表,1997(10):1-3。

[7] BGND. 《一文读懂磁传感器》. 《传感器与集成电路》 http://www.360doc.com/content/16/1027/11/36367270_60175 8627.shtml 2016

[8] Mcguire T R, Potter R I. Anisotropic magnetoresistance in ferromagnetic $3 \mathrm{~d}$ alloys[J]. IEEE Transactions on Magnetics, 1975, 11(4):1018-1038.

[9] Binasch G, Grünberg P, Saurenbach F, et al. Enhanced magnetoresistance in layered magnetic structures with antiferromagnetic interlayer exchange.[J]. Phys Rev B Condens Matter, 1989, 39(7):4828-4830.
[10] Julliere, M., Tunneling between ferromagnetic films, Phys. Lett., 1975, 54A(3): 225.

[11] Ikeda S, Hayakawa J, Ashizawa Y, et al. Tunnel magnetoresistance of $604 \%$ at $300 \mathrm{~K}$ by suppression of $\mathrm{Ta}$ diffusion in $\mathrm{CoFeB} / \mathrm{MgO} / \mathrm{CoFeB}$ pseudo-spin-valves annealed at high temperature[J]. Applied Physics Letters, 2008, 93(8):L588.

[12] Thomas H P. Direction responsive system: US, US2016977[P]. 1935.

[13] Ripka P. Advances in fluxgate sensors[J]. Sensors \& Actuators A Physical, 2003, 106(1-3):8-14.

[14] Hwang J S, Park H S, Shim D S, et al. Electronic compass using two-axis micro fluxgate sensing element[C]// Transducers, Solid-State Sensors, Actuators and Microsystems, International Conference on. IEEE, 2003:1618-1621 vol.2.

[15] Nielsen O V, Brauer P, Primdahl F, et al. A high-precision triaxial fluxgate sensor for space applications: layout and choice of materials[J]. Sensors \& Actuators A Physical, 1997, 59(1-3):168-176.

[16] 郭博.硅基微型磁通门传感器关键技术研究[D].西北工业大 学, 2015。

[17] Choi S O, Kawahito S, Matsumoto Y, et al. An integrated micro fluxgate magnetic sensor[J]. Sensors \& Actuators A Physical, 1996, 55(2-3):121-126.

[18] 南策文.多铁性材料研究进展及发展方向[J].中国科学:技术 科学, 2015(4):339-357。

[19] Wang K F, Liu J M, Ren Z F. ChemInform Abstract: Multiferroicity - The Coupling Between Magnetic and Polarization Orders[J]. Cheminform, 2010, 41(31):no-no.

[20] 甘蹈.离子束溅射制备FeGa薄膜及其磁传感性能研究 $[D]$.上 海师范大学,2015。

[21] Pan D A, Bai Y, Chu W Y, et al. Ni-PZT-Ni trilayered magnetoelectric composites synthesized by electro-deposition. J Phys Condens Mat, 2008, 20: 025203.

[22] Greve H, Woltermann E, Quenzer H J, et al. Giant magnetoelectric coefficients in (Fe90Co10)78Si12B10-AlN thin film composites. Appl PhysLett, 2010, 96: 182501.

[23] 冯春鹏.多铁纳米MEMS传感器性能测试系统设计[D].北方 工业大学2017。

[24] 李扩社,李红卫,严辉, 等. 磁电复合材料的研究进展 $[\mathrm{J}]$ 稀有 金属,2008,32(3):369-374。

[25] Ryu J, Carazo A V, Uchino K, et al. Magnetoelectric properties in piezoelectric and magnetostrictive laminate composites. Japanese Journal of Applied Physics Part 1-Regular Papers Short Notes \& Review Papers, 2001, 40(8): 4948-4951.

[26] Nan C W, Bichurin M I, Dong S X, et al. Multiferroic magnetoelectric composites: Historical perspective, status, and future directions. JAppl Phys, 2008, 103: 031101.

[27] 马静,施展,林元华,等.准2-2型磁电多层复合材料的磁电性 能[J].物理学报, 2009, 58(8):5852-5856。 